TITLE:

\title{
Kinetic theory of point vortex systems from the Bogoliubov-Born- Green-Kirkwood-Yvon hierarchy
}

\section{$\operatorname{AUTHOR}(S)$ :}

Sano, Mitsusada M.

\section{CITATION:}

Sano, Mitsusada M.. Kinetic theory of point vortex systems from the Bogoliubov-BornGreen-Kirkwood-Yvon hierarchy. Physical Review E 2007, 76(4(Part 2)): 046312.

\section{ISSUE DATE:}

2007-10

URL:

http://hdl.handle.net/2433/59277

RIGHT:

Copyright 2007 American Physical Society 
PHYSICAL REVIEW E 76, 046312 (2007)

\title{
Kinetic theory of point vortex systems from the Bogoliubov-Born-Green-Kirkwood-Yvon hierarchy
}

\author{
Mitsusada M. Sano \\ Graduate School of Human and Environmental Studies, Kyoto University, Sakyo, Kyoto 606-8501, Japan
}

(Received 19 April 2007; revised manuscript received 20 June 2007; published 12 October 2007)

\begin{abstract}
Kinetic equations are derived from the Bogoliubov-Born-Green-Kirkwood-Yvon hierarchy for point vortex systems in an infinite plane. At the level of approximation for the Landau equation, the collision term of the kinetic equation derived coincides with that by Chavanis [Phys. Rev. E 64, 026309 (2001)]. Furthermore, we derive a kinetic equation corresponding to the Balescu-Lenard equation for plasmas using the theory of the Fredholm integral equation. For large $N$, this kinetic equation is reduced to the Landau equation above.
\end{abstract}

DOI: 10.1103/PhysRevE.76.046312

PACS number(s): 47.32.C-, 05.20.Dd, 05.20.Jj, 05.20.-y

\section{INTRODUCTION}

A point vortex system is a model of continuous twodimensional (2D) inviscid fluid dynamics. In fact, a point vortex is an idealized vortex of real vortex in 2D fluids. However, the point vortex system carries some important properties of continuous $2 \mathrm{D}$ inviscid fluid dynamics $[1,2]$. It is formulated as a Hamilton dynamical system $[3,4]$. Therefore, a standard statistical mechanical theory was developed by Onsager [5]. In his formulation, states of a point vortex system are classified into two categories: one is positive temperature states, and the other is negative temperature states. In the positive temperature states, point vortices are distributed in a scattered way. However, in the negative temperature states, huge vortices are formed in time evolution. The negative temperature states are phenomena observed in Earth's surface - that is, the formation of huge vortices, such as hurricanes and typhoons. Onsager's ideas are recently reviewed by Eyink and Sreenivasan [6]. Since Onsager, researchers have considered the equilibrium statistical mechanics of point vortex systems. The main purpose of their studies is to construct the equilibrium states of a point vortex system [7-12]. The Poisson-Boltzmann equation is used to obtain the equilibrium states. For some cases, the equilibrium states are analytically obtained [13].

The next stage of the statistical mechanics of point vortex systems focuses on nonequilibrium properties. Standard nonequilibrium statistical mechanics goes toward kinetic theory. Two authors have studied kinetic theory of point vortex system: i.e., Marmanis [14] and Chavanis [15]. Marmanis considered a gas of binary pairs of positive and negative point vortices. Chavanis considered a gas of point vortices with the same circulation. In this paper, we are interested in Chavanis's results.

The system considered by Chavanis is closely related to the system of non-neutral plasmas in the Malmberg trap [16-22]. The dynamics of the non-neutral plasma in the Malmberg trap is described by the 2D Euler equation in a circular domain, using the guiding center approximation. Thus, if we are interested in non-neutral plasma in the experimental situation, as an idealized model, we should consider a point vortex system in a circular domain. From experiments on non-neutral plasmas, many interesting properties of vortex dynamics of the $2 \mathrm{D}$ Euler equation are now known [16-22]: (i) diocotron instability (i.e., in other words, Kelvin-Helmholtz instability), (ii) violent relaxation, (iii) slow decay, (iv) vortex crystals, and (v) merger of vortices. As a theoretical aspect, recently the slow decay was numerically analyzed using the point vortex system [23-25]. Although there is a difference in the boundary condition, these properties introduced here are common in the point vortex systems in an infinite plane.

Back to the point vortex systems in an infinite plane, Chavanis derived serveral kinetic equations for the point vortex system in an infinite plane, in which the point vortices have the same circulation, and estimated interesting physical quantities, like the diffusion coefficient and the drift term, by using his kinetic equations $[15,26,27]$. In this paper, we develop a kinetic theory through the Bogoliubov-Born-GreenKirkwood-Yvon (BBGKY) hierarchy [28-31] (for the text, see Ref. [32]). We compare our theory with that by Chavanis. The main purposes of this paper is as follows: (i) We derive a kinetic equation (i.e., the Landau equation), which is identical to that by Chavanis [15,27]. (ii) Futhermore, we derive a kinetic equation (i.e., the Balescu-Lenard equation), which includes more correlation-i.e., the collective effects. (iii) We show that for large $N$, this kinetic equation is reduced to the Landau equation.

The organization of this paper is as follows. In Sec. II, the equations of motion for a point vortex system in an infinite plane are presented. In Sec. III, the treatment of the BBGKY hierarchy is shown. Two key equations for time evolution of the distribution function and the correlation function are derived. Using these equations, we derive the Vlasov equation, the Landau equation and the Balescu-Lenard equation for point vortex system in an infinite plane. It is shown that for large $N$, the Balescu-Lenard equation is reduced to the Landau equation. In Sec. IV, we summarize the results of this paper and give future problems.

\section{EQUATIONS OF MOTION}

Let us consider a point vortex system, which consists of $N$ point vortices with the same circulation $\gamma$ in an infinite plane. The Hamiltonian of this system is given by 


$$
H=-\frac{\gamma^{2}}{4 \pi} \sum_{i \neq j}^{N} \ln \left|\mathbf{r}_{i}-\mathbf{r}_{j}\right|,
$$

where $\mathbf{r}_{i}=\left(x_{i}, y_{i}\right)$. The equations of motion are written by using the Hamiltonian:

$$
\gamma \frac{d x_{i}}{d t}=\frac{\partial H}{\partial y_{i}}, \gamma \frac{d y_{i}}{d t}=-\frac{\partial H}{\partial x_{i}} .
$$

Thus, the velocities of the $i$ th point vortex in the $x$ and $y$ directions are given by

$$
\begin{gathered}
v_{i}^{(x)}=\frac{\gamma}{2 \pi} \sum_{j \neq i}^{N} \frac{\left(\mathbf{r}_{i}-\mathbf{r}_{j}\right)_{y}}{\left|\mathbf{r}_{i}-\mathbf{r}_{j}\right|^{2}}, \\
v_{i}^{(y)}=-\frac{\gamma}{2 \pi} \sum_{j \neq i}^{N} \frac{\left(\mathbf{r}_{i}-\mathbf{r}_{j}\right)_{x}}{\left|\mathbf{r}_{i}-\mathbf{r}_{j}\right|^{2}} .
\end{gathered}
$$

It is convenient to rewrite the velocity in the following form:

$$
\mathbf{v}_{i}=\sum_{j \neq i} \mathbf{v}(j \rightarrow i)
$$

where

$$
\mathbf{v}(j \rightarrow i)=-\frac{\gamma}{2 \pi} \mathbf{z} \times \frac{\mathbf{r}_{i}-\mathbf{r}_{j}}{\left|\mathbf{r}_{i}-\mathbf{r}_{j}\right|^{2}}=\frac{\gamma}{2 \pi} \frac{1}{\left|\mathbf{r}_{i}-\mathbf{r}_{j}\right|^{2}} J \cdot \mathbf{r}_{i j},
$$

$J$ is the $2 \times 2$ symplectic matrix

$$
J=\left(\begin{array}{cc}
0 & 1 \\
-1 & 0
\end{array}\right)
$$

$\mathbf{z}$ is the unit vector along the $z$ axis, and $\mathbf{r}_{i j}=\mathbf{r}_{i}-\mathbf{r}_{j}$. This system has several conserved quantities: (i) energy-i.e., $H$ $=E$; (ii) angular impulse-i.e., $I=\gamma \sum_{i=1}^{N}\left|\mathbf{r}_{i}\right|^{2}$; (iii) linear impulse-i.e., $\mathbf{L}=\gamma \sum_{i=1}^{N} \mathbf{r}_{i}$.

Now we define the vorticity $\omega(\mathbf{r} ; t)$ :

$$
\omega(\mathbf{r} ; t)=\sum_{i=1}^{N} \gamma \delta\left(\mathbf{r}-\mathbf{r}_{i}\right)
$$

and the stream function $\psi(\mathbf{r} ; t)$,

$$
\psi(\mathbf{r} ; t)=-\frac{\gamma}{2 \pi} \sum_{i=1}^{N} \ln \left|\mathbf{r}-\mathbf{r}_{i}\right| .
$$

Using the stream function, the velocity of the $i$ th point vortex is given by

$$
\mathbf{v}_{i}=-\mathbf{z} \times \nabla \psi\left(\mathbf{r}=\mathbf{r}_{i} ; t\right)=J \cdot \nabla \psi\left(\mathbf{r}=\mathbf{r}_{i} ; t\right) .
$$

It is easily confirmed that the vorticity satisfies the twodimensional Euler equation

$$
\frac{\partial \omega}{\partial t}+\mathbf{v} \cdot \nabla \omega=0
$$

\section{BBGKY HIERARCHY}

We define the distribution function of $N$-point vortex systems:

$$
F=F\left(\mathbf{r}_{1}, \mathbf{r}_{2}, \ldots, \mathbf{r}_{N} ; t\right) .
$$

The Liouvile equation for $N$-point vortex systems in an infinite plane is given by

$$
\frac{\partial F}{\partial t}=\mathcal{L} F
$$

where

$$
\mathcal{L}=\sum_{i=1}^{N} \frac{1}{\gamma}\left(\frac{\partial H}{\partial x_{i}} \frac{\partial}{\partial y_{i}}-\frac{\partial H}{\partial y_{i}} \frac{\partial}{\partial x_{i}}\right) .
$$

The Liouvillian $\mathcal{L}$ is rewritten as follows:

$$
\begin{aligned}
\mathcal{L} & =-\sum_{i=1}^{N} \sum_{j \neq i}\left(v_{x}(j \rightarrow i) \frac{\partial}{\partial y_{i}}+v_{y}(j \rightarrow i) \frac{\partial}{\partial x_{i}}\right) \\
& =-\sum_{i<j}^{N} \mathbf{v}(j \rightarrow i) \cdot \nabla_{i j},
\end{aligned}
$$

where $\boldsymbol{\nabla}_{i}=\frac{\partial}{\partial \mathbf{r}_{i}}$ and $\boldsymbol{\nabla}_{i j}=\boldsymbol{\nabla}_{i}-\boldsymbol{\nabla}_{j}$. Now we have used the fact that $\mathbf{v}(j \rightarrow i)=-\mathbf{v}(i \rightarrow j)$. We define $\mathcal{L}_{i j}$ as

$$
\begin{aligned}
\mathcal{L}_{i j} & =-\mathbf{v}(j \rightarrow i) \cdot \boldsymbol{\nabla}_{i j} \\
& =-\mathbf{v}(j \rightarrow i) \cdot \boldsymbol{\nabla}_{i}-\mathbf{v}(i \rightarrow j) \cdot \boldsymbol{\nabla}_{j} .
\end{aligned}
$$

Thus, the Liouvillian becomes

$$
\mathcal{L}=\sum_{i<j}^{N} \mathcal{L}_{i j} .
$$

We define the $s$-body reduced distribution function

$$
\begin{aligned}
f_{s}\left(\mathbf{r}_{1}, \ldots, \mathbf{r}_{s}\right)= & \frac{N !}{(N-s) !} \int d \mathbf{r}_{s+1} d \mathbf{r}_{s+2} \ldots d \mathbf{r}_{N} \\
& \times F\left(\mathbf{r}_{1}, \ldots, \mathbf{r}_{s}, \mathbf{r}_{s+1}, \ldots, \mathbf{r}_{N}\right) .
\end{aligned}
$$

Carrying out a usual manipulation of the BBGKY hierarchy (see [32] for details), we obtain the following time-evolution equations for the $s$-body reduced distribution function $f_{s}\left(\mathbf{r}_{1}, \ldots, \mathbf{r}_{s}\right)$ :

$$
\partial_{t} f_{0}=0
$$

and

$$
\begin{aligned}
\partial_{t} f_{s}\left(\mathbf{r}_{1}, \ldots, \mathbf{r}_{s}\right)= & \sum_{i<j} \mathcal{L}_{i j} f_{s}\left(\mathbf{r}_{1}, \ldots, \mathbf{r}_{s}\right) \\
& +\sum_{i=1}^{s} \int d \mathbf{r}_{s+1} \mathcal{L}_{i, s+1} f_{s+1}\left(\mathbf{r}_{1}, \ldots, \mathbf{r}_{s+1}\right) .
\end{aligned}
$$

This is the BBGKY hierarchy for the $s$-body reduced distribution functions. The time evolution of the $s$-body reduced distribution function is determined by the $s$-body reduced distribution function and the $(s+1)$-body reduced distribution function.

For $s=1$, we have 


$$
\partial_{t} f_{1}\left(\mathbf{r}_{1}\right)=\int d \mathbf{r}_{2} \mathcal{L}_{12} f_{2}\left(\mathbf{r}_{1}, \mathbf{r}_{2}\right) .
$$

Now we use the two-body and three-body correlation functions $g_{2}\left(\mathbf{r}_{1}, \mathbf{r}_{2}\right)$ and $g_{3}\left(\mathbf{r}_{1}, \mathbf{r}_{2}, \mathbf{r}_{3}\right)$. The two- and three-body correlation functions are related to the two- and three-body reduced distribution functions as follows:

$$
\begin{aligned}
& f_{2}\left(\mathbf{r}_{1}, \mathbf{r}_{2}\right)=f_{1}\left(\mathbf{r}_{1}\right) f_{1}\left(\mathbf{r}_{2}\right)+g_{2}\left(\mathbf{r}_{1}, \mathbf{r}_{2}\right), \\
& f_{3}\left(\mathbf{r}_{1}, \mathbf{r}_{2}, \mathbf{r}_{3}\right)= f_{1}\left(\mathbf{r}_{1}\right) f_{1}\left(\mathbf{r}_{2}\right) f_{1}\left(\mathbf{r}_{3}\right)+f_{1}\left(\mathbf{r}_{1}\right) g_{2}\left(\mathbf{r}_{2}, \mathbf{r}_{3}\right) \\
&+f_{1}\left(\mathbf{r}_{2}\right) g_{2}\left(\mathbf{r}_{1}, \mathbf{r}_{3}\right)+f_{1}\left(\mathbf{r}_{3}\right) g_{2}\left(\mathbf{r}_{1}, \mathbf{r}_{2}\right) \\
&+g_{3}\left(\mathbf{r}_{1}, \mathbf{r}_{2}, \mathbf{r}_{3}\right) .
\end{aligned}
$$

Thus, the correlation function describes the deviation from the product of the reduced distribution functions. Inserting Eq. (22) into Eq. (21), we obtain

$$
\partial_{t} f_{1}\left(\mathbf{r}_{1}\right)=\int d \mathbf{r}_{2}\left[\mathcal{L}_{12} f_{1}\left(\mathbf{r}_{1}\right) f_{1}\left(\mathbf{r}_{2}\right)+\mathcal{L}_{12} g_{2}\left(\mathbf{r}_{1}, \mathbf{r}_{2}\right)\right] .
$$

Inserting Eqs. (22) and (23) into Eq. (20), the time-evolution equation of $f_{2}\left(\mathbf{r}_{1}, \mathbf{r}_{2}\right)$ is obtained:

$$
\begin{aligned}
\partial_{t} f_{2}\left(\mathbf{r}_{1}, \mathbf{r}_{2}\right)= & \mathcal{L}_{12}\left[f_{1}\left(\mathbf{r}_{1}\right) f_{1}\left(\mathbf{r}_{2}\right)+g_{2}\left(\mathbf{r}_{1}, \mathbf{r}_{2}\right)\right] \\
& +\int d \mathbf{r}_{3}\left\{\mathcal { L } _ { 1 3 } \left[f_{1}\left(\mathbf{r}_{1}\right) f_{1}\left(\mathbf{r}_{2}\right) f_{1}\left(\mathbf{r}_{3}\right)\right.\right. \\
& +f_{1}\left(\mathbf{r}_{1}\right) g_{2}\left(\mathbf{r}_{2}, \mathbf{r}_{3}\right)+f_{1}\left(\mathbf{r}_{2}\right) g_{2}\left(\mathbf{r}_{1}, \mathbf{r}_{3}\right) \\
& \left.\left.+f_{1}\left(\mathbf{r}_{3}\right) g_{2}\left(\mathbf{r}_{1}, \mathbf{r}_{2}\right)+g_{3}\left(\mathbf{r}_{1}, \mathbf{r}_{2}, \mathbf{r}_{3}\right)\right]+(1 \Leftrightarrow 2)\right\} .
\end{aligned}
$$

Similarly, using Eq. (24), inserting Eq. (22) into Eq. (25), and manipulating the resulting equations, the time-evolution equation of $g_{2}\left(\mathbf{r}_{1}, \mathbf{r}_{2}\right)$ is obtained:

$$
\begin{aligned}
\partial_{t} g_{2}\left(\mathbf{r}_{1}, \mathbf{r}_{2}\right)= & \mathcal{L}_{12} f_{1}\left(\mathbf{r}_{1}\right) f_{1}\left(\mathbf{r}_{2}\right)+\mathcal{L}_{12} g_{2}\left(\mathbf{r}_{1}, \mathbf{r}_{2}\right) \\
& +\int d \mathbf{r}_{3}\left\{\mathcal{L}_{13} f_{1}\left(\mathbf{r}_{1}\right) g_{2}\left(\mathbf{r}_{2}, \mathbf{r}_{3}\right)\right. \\
& +\mathcal{L}_{23} f_{1}\left(\mathbf{r}_{2}\right) g_{2}\left(\mathbf{r}_{1}, \mathbf{r}_{3}\right)+\left(\mathcal{L}_{13}+\mathcal{L}_{23}\right) \\
& \left.\times\left[f_{1}\left(\mathbf{r}_{3}\right) g_{2}\left(\mathbf{r}_{1}, \mathbf{r}_{2}\right)+g_{3}\left(\mathbf{r}_{1}, \mathbf{r}_{2}, \mathbf{r}_{3}\right)\right]\right\} .
\end{aligned}
$$

Equation (26) is slightly different from the recent result by Chavanis [27], who also developed a BBGKY hierarchy for the point vortex gas. The reason for these differences is unknown.

Following Ref. [27], we shall close the hiearchy of BBGKY equations by considering an expansion in powers of $1 / N$ for $N \rightarrow+\infty$. In the large $N$-limit-i.e., the hydrodynamic limit-we should preserve the total circulation $\Gamma$. Thus, the circulation $\gamma$ is $\gamma=\Gamma / N$, where $\Gamma=$ const. The order estimate of each function is $f \sim 1, g_{2} \sim 1 / N, \gamma \sim 1 / N$, and $\mathcal{L}_{i j} \sim 1 / N$. Thus, in Eq. (26), the first term is $\sim 1 / N$. The second term is $\sim 1 / N^{2}$. The integral part is $\sim 1 / N$, since the integration over $\mathbf{r}_{3}$ gives an $N$ factor. The term including the term $g_{3}$ is $\sim 1 / N^{2}$. In the following treatment, the function $g_{3}$ is omitted, since we cut the correlation-i.e., truncate a chain of the BBGKY hierarchy.

\section{A. Vlasov equation}

A mean-field approximation is performed by neglecting the term of the correlation function in Eq. (24):

$$
\partial_{t} f\left(\mathbf{r}_{1}\right)=\int d \mathbf{r}_{2} \mathcal{L}_{12} f\left(\mathbf{r}_{1}\right) f\left(\mathbf{r}_{2}\right)
$$

Using Eq. (16), we obtain

$$
\frac{\partial f\left(\mathbf{r}_{1}\right)}{\partial t}+\left\langle\mathbf{v}_{1}\right\rangle \cdot \nabla_{1} f\left(\mathbf{r}_{1}\right)=0
$$

where

$$
\left\langle\mathbf{v}_{1}\right\rangle=\int d \mathbf{r}_{2} f\left(\mathbf{r}_{2}\right)\left[v_{x}(2 \rightarrow 1) \mathbf{i}+v_{y}(2 \rightarrow 1) \mathbf{j}\right] .
$$

This is a mean-field equation for the point vortex system in an infinite plane. It is analogous to the Vlasov equation in plasma physics and in stellar dynamics. We should note that this equation (28) is nothing but the 2D Euler equation.

\section{B. Landau equation}

The next-higher-order approximation is started with Eq. (24) preserving the correlation function

$$
\partial_{t} f\left(\mathbf{r}_{1}\right)=\int d \mathbf{r}_{2}\left[\mathcal{L}_{12} f\left(\mathbf{r}_{1}\right) f\left(\mathbf{r}_{2}\right)+\mathcal{L}_{12} g_{2}\left(\mathbf{r}_{1}, \mathbf{r}_{2}\right)\right] .
$$

The right-hand-side of Eq. (30) is upto the order $1 / N^{2}$. For the correlation function $g_{2}\left(\mathbf{r}_{1}, \mathbf{r}_{2}\right)$, we approximate Eq. (26) upto the order $1 / N$ :

$$
\begin{aligned}
\partial_{t} g_{2}\left(\mathbf{r}_{1}, \mathbf{r}_{2}\right)= & \mathcal{L}_{12} f\left(\mathbf{r}_{1}\right) f\left(\mathbf{r}_{2}\right)+\int d \mathbf{r}_{3}\left(\mathcal{L}_{13}+\mathcal{L}_{23}\right) f\left(\mathbf{r}_{3}\right) g_{2}\left(\mathbf{r}_{1}, \mathbf{r}_{2}\right) \\
= & \mathcal{L}_{12} f\left(\mathbf{r}_{1}\right) f\left(\mathbf{r}_{2}\right)+\left(-\left\langle\mathbf{v}_{1}\right\rangle \cdot \nabla_{1}-\left\langle\mathbf{v}_{2}\right\rangle \cdot \nabla_{2}\right) \\
& \times g_{2}\left(\mathbf{r}_{1}, \mathbf{r}_{2}\right) .
\end{aligned}
$$

Therefore, the correlation function is advected by $\left\langle\mathbf{v}_{1}\right\rangle$ and $\left\langle\mathbf{v}_{2}\right\rangle$. Equation (31) is formally solved as

$$
\begin{aligned}
g_{2}\left(\mathbf{r}_{1}, \mathbf{r}_{2} ; t\right)= & U_{12}(t) g_{2}\left(\mathbf{r}_{1}, \mathbf{r}_{2} ; 0\right)+\int_{0}^{t} d \tau U_{12}(\tau) \mathcal{L}_{12} f\left(\mathbf{r}_{1} ; t-\tau\right) \\
& \times f\left(\mathbf{r}_{2} ; t-\tau\right),
\end{aligned}
$$

where

$$
U_{12}(\tau)=\exp \left[-\int_{0}^{\tau} d t^{\prime}\left\langle\mathbf{v}_{1}\right\rangle \cdot \nabla_{1}-\int_{0}^{\tau} d t^{\prime}\left\langle\mathbf{v}_{2}\right\rangle \cdot \nabla_{2}\right] .
$$

Inserting Eq. (32) into Eq. (30), we obtain

$$
\begin{aligned}
\partial_{t} f\left(\mathbf{r}_{1}\right)= & \int d \mathbf{r}_{2} \mathcal{L}_{12} f\left(\mathbf{r}_{1}\right) f\left(\mathbf{r}_{2}\right)+\int d \mathbf{r}_{2} \mathcal{L}_{12} U_{12}(t) g_{2}\left(\mathbf{r}_{1}, \mathbf{r}_{2} ; 0\right) \\
& +\int d \mathbf{r}_{2} \int_{0}^{t} d \tau \mathcal{L}_{12} U_{12}(\tau) \mathcal{L}_{12} f\left(\mathbf{r}_{1} ; t-\tau\right) f\left(\mathbf{r}_{2} ; t-\tau\right) .
\end{aligned}
$$

On the right-hand side of Eq. (34), the second term vanishes 
for large $t$ (i.e., the correlation decays); thus, we have

$$
\begin{aligned}
\partial_{t} f\left(\mathbf{r}_{1}\right)= & \int d \mathbf{r}_{2} \mathcal{L}_{12} f\left(\mathbf{r}_{1}\right) f\left(\mathbf{r}_{2}\right) \\
& +\int d \mathbf{r}_{2} \int_{0}^{t} d \tau \mathcal{L}_{12} U_{12}(\tau) \mathcal{L}_{12} f\left(\mathbf{r}_{1} ; t-\tau\right) f\left(\mathbf{r}_{2} ; t-\tau\right) .
\end{aligned}
$$

We have to evaluate the following term:

$$
\mathcal{K}_{\mathrm{coll}}^{(L)}\{f f\}=\int d \mathbf{r}_{2} \int_{0}^{t} d \tau \mathcal{L}_{12} U_{12}(\tau) \mathcal{L}_{12} f\left(\mathbf{r}_{1} ; t-\tau\right) f\left(\mathbf{r}_{2} ; t-\tau\right) .
$$

Using the approximation

$$
f\left(\mathbf{r}_{1} ; t-\tau\right) f\left(\mathbf{r}_{2} ; t-\tau\right) \approx U_{12}(-\tau) f\left(\mathbf{r}_{1} ; t\right) f\left(\mathbf{r}_{2} ; t\right),
$$

we obtain

$$
\begin{aligned}
\mathcal{K}_{\mathrm{coll}}^{(L)}\{f f\} & \approx \int d \mathbf{r}_{2} \int_{0}^{t} d \tau \mathcal{L}_{12} U_{12}(\tau) \mathcal{L}_{12} U_{12}(-\tau) f\left(\mathbf{r}_{1} ; t\right) f\left(\mathbf{r}_{2} ; t\right) \\
& =\nabla_{1} \cdot \int d \mathbf{r}_{2} \int_{0}^{t} d \tau \mathbf{v}_{1}(t) \mathbf{v}_{1}(t-\tau) \nabla_{12} f\left(\mathbf{r}_{1} ; t\right) f\left(\mathbf{r}_{2} ; t\right)
\end{aligned}
$$

The kinetic equation obtained here is

$$
\begin{aligned}
\frac{\partial f\left(\mathbf{r}_{1}\right)}{\partial t}+\left\langle\mathbf{v}_{1}\right\rangle \cdot \nabla_{1} f\left(\mathbf{r}_{1}\right)= & \nabla_{1} \cdot \int d \mathbf{r}_{2} \int_{0}^{t} d \tau \mathbf{v}_{1}(t) \mathbf{v}_{1}(t-\tau) \cdot \nabla_{12} \\
& \times f\left(\mathbf{r}_{1} ; t\right) f\left(\mathbf{r}_{2} ; t\right)
\end{aligned}
$$

where $\mathbf{v}_{1}(t)$ is advected as $\mathbf{v}_{1}(t-\tau)=U_{12}(\tau) \mathbf{v}_{1}(t) U_{12}(-\tau)$ and $\mathbf{r}_{i}(t-\tau)=\mathbf{r}_{i}(t)-\int_{0}^{\tau} d t^{\prime}\left\langle\mathbf{v}_{i}\right\rangle\left(\mathbf{r}_{i}\left(t-t^{\prime}\right), t-t^{\prime}\right)$. This equation is analogous to the Landau equation in plasma physics and in stellar dynamics. This equation coincides with the result of $[15,27]$. As shown in Ref. [15], this equation conserves the angular impulse and the linear impulse. If we use the Markovianization-i.e., extending the time integral to infinity-we obtain

$$
\mathcal{K}_{\mathrm{coll}}^{(L)}\{f f\} \boldsymbol{\nabla}_{1} \cdot \int d \mathbf{r}_{2} \int_{0}^{\infty} d \tau \mathbf{v}_{1}(t) \mathbf{v}_{1}(t-\tau) \cdot \boldsymbol{\nabla}_{12} f\left(\mathbf{r}_{1} ; t\right) f\left(\mathbf{r}_{2} ; t\right) .
$$

However, it is not known whether the Markovianization is assured or not, since point vortex dynamics sometimes gives long-time tail: i.e., the strong correlation. In particular, in Ref. [24], it is shown that the diffusion process for the point vortex exhibits Lévy flight.

Chavanis estimated the relaxation time $t_{\text {relax }}$ by using the estimate of the diffusion coefficient [15] as $t_{\text {relax }}$ $\sim N /(\ln N) t_{D}$, where the dynamical time is $t_{D} \sim\langle\omega\rangle^{-1}$ $\sim R^{2} / \Gamma$, which is the time determined by the mean rotation time, and $R$ is the size of the vortex. His estimate of Ref. [15] would be incorrect. In the kinetic theory, the $N$ dependence of $t_{\text {relax }}$ is determined by the $N$ dependence of the collision term: i.e., $\mathcal{K}_{\text {coll }}^{(L)} \sim O(1 / N)$. This gives $t_{\text {relax }} \sim N t_{D}$. Recently
Chavanis and Lemou used this estimate [26,27]. This estimate is consistent with the numerical result by Kawahara and Nakanishi for the system in a circular domain [24].

\section{Balescu-Lenard equation}

In this subsection, we derive a kinetic equation for point vortex systems in an infinite plane, which is analogous to the Balescu-Lenard equation in plasma physics. The starting point is the time-evolution equations of the one-body reduced distribution function $f\left(\mathbf{r}_{1}\right)$ and the two-body correlation function $g_{2}\left(\mathbf{r}_{1}, \mathbf{r}_{2}\right)$ :

$$
\partial_{t} f\left(\mathbf{r}_{1}\right)=\int d \mathbf{r}_{2}\left[\mathcal{L}_{12} f\left(\mathbf{r}_{1}\right) f\left(\mathbf{r}_{2}\right)+\mathcal{L}_{12} g_{2}\left(\mathbf{r}_{1}, \mathbf{r}_{2}\right)\right],
$$

and

$$
\begin{aligned}
\partial_{t} g_{2}\left(\mathbf{r}_{1}, \mathbf{r}_{2}\right)= & \mathcal{L}_{12} f\left(\mathbf{r}_{1}\right) f\left(\mathbf{r}_{2}\right)+\mathcal{L}_{12} g_{2}\left(\mathbf{r}_{1}, \mathbf{r}_{2}\right) \\
& +\int d \mathbf{r}_{3}\left\{\mathcal{L}_{13} f\left(\mathbf{r}_{1}\right) g_{2}\left(\mathbf{r}_{2}, \mathbf{r}_{3}\right)+\mathcal{L}_{23} f\left(\mathbf{r}_{2}\right) g_{2}\left(\mathbf{r}_{1}, \mathbf{r}_{3}\right)\right. \\
& \left.+\left(\mathcal{L}_{13}+\mathcal{L}_{23}\right)\left[f\left(\mathbf{r}_{3}\right) g_{2}\left(\mathbf{r}_{1}, \mathbf{r}_{2}\right)\right]\right\} .
\end{aligned}
$$

The two-body correlation function is formally solved as

$$
\begin{aligned}
g_{2}\left(\mathbf{r}_{1}, \mathbf{r}_{2} ; t\right)= & \int_{0}^{t} d \tau U_{12}(\tau)\left\{\mathcal{L}_{12} U_{12}(-\tau) f\left(\mathbf{r}_{1}\right) f\left(\mathbf{r}_{2}\right)\right. \\
& +\mathcal{L}_{12} U_{12}(-\tau) g_{2}\left(\mathbf{r}_{1}, \mathbf{r}_{2}\right) \\
& +\int d \mathbf{r}_{3}\left[\mathcal{L}_{13} U_{12}(-\tau) f\left(\mathbf{r}_{1}\right) g_{2}\left(\mathbf{r}_{2}, \mathbf{r}_{3}\right)\right. \\
& \left.\left.+\mathcal{L}_{23} U_{12}(-\tau) f\left(\mathbf{r}_{2}\right) g_{2}\left(\mathbf{r}_{1}, \mathbf{r}_{3}\right)\right]\right\}
\end{aligned}
$$

The kinetic equation is formally obtained as

$$
\frac{\partial f_{1}}{\partial t}+\left\langle\mathbf{v}_{1}\right\rangle \cdot \nabla_{1} f_{1}=\int d \mathbf{r}_{2} \mathcal{L}_{12} g_{2}\left(\mathbf{r}_{1}, \mathbf{r}_{2} ; t\right) .
$$

If, as done for plasma systems in Ref. [32], we set

$$
g_{2}\left(\mathbf{r}_{1}, \mathbf{r}_{2} ; t\right)=g_{2}\left(\mathbf{r}_{1}-\mathbf{r}_{2} ; t\right)=\int d \mathbf{k} \exp \left[i \mathbf{k} \cdot\left(\mathbf{r}_{1}-\mathbf{r}_{2}\right)\right] \widetilde{g}_{2}(\mathbf{k} ; t),
$$

the right-hand side of Eq.(44) - i.e., the collision termvanishes:

$$
\begin{aligned}
\int d \mathbf{r}_{2} \mathcal{L}_{12} g_{2}\left(\mathbf{r}_{1}, \mathbf{r}_{2} ; t\right) \\
=\frac{1}{\gamma} \int d \mathbf{r}_{2}\left[\nabla_{1} V\left(\mathbf{r}_{1}-\mathbf{r}_{2}\right)\right]^{\top} \cdot J \cdot \nabla_{1} g_{2}\left(\mathbf{r}_{1}, \mathbf{r}_{2}\right) \\
=\frac{1}{\gamma} \int d \mathbf{r}_{2} \int d \mathbf{k} \exp \left[i \mathbf{k} \cdot\left(\mathbf{r}_{1}-\mathbf{r}_{2}\right)\right] \widetilde{V}(k)(i \mathbf{k})^{\top} \cdot J \nabla_{1} \\
\quad \times \int d \mathbf{k}^{\prime} \exp \left[i \mathbf{k}^{\prime} \cdot\left(\mathbf{r}_{1}-\mathbf{r}_{2}\right)\right] \widetilde{g}_{2}\left(\mathbf{k}^{\prime} ; t\right) \\
=\frac{(2 \pi)^{2}}{\gamma} \int d \mathbf{k} \tilde{V}(k) \mathbf{k}^{\top} \cdot J \cdot \mathbf{k} \widetilde{g}_{2}(-\mathbf{k} ; t)=0
\end{aligned}
$$


since $\mathbf{k}^{\top} \cdot J \cdot \mathbf{k}=0$. Here " $\mathbf{A}^{\top}$ " means the transpose of the vector A. In Eq. (45), the homogeneity is assumed. The above result shows that inhomogeneity is important for point vortex systems. To not make the collision term vanish, we change the definition of the Fourier transform of $g_{2}\left(\mathbf{r}_{1}, \mathbf{r}_{2} ; t\right)$. Therefore, we set

$g_{2}\left(\mathbf{r}_{1}, \mathbf{r}_{2} ; t\right)=\int d \mathbf{k}_{1} \int d \mathbf{k}_{2} \exp \left[i \mathbf{k}_{1} \cdot \mathbf{r}_{1}+i \mathbf{k}_{2} \cdot \mathbf{r}_{2}\right] \widetilde{g}_{2}\left(\mathbf{k}_{1}, \mathbf{k}_{2} ; t\right)$.

$\mathcal{L}_{12}$ can be rewritten in the form

$$
\mathcal{L}_{12}=\frac{1}{\gamma}\left[\nabla_{1} V\left(\mathbf{r}_{1}-\mathbf{r}_{2}\right)\right]^{\top} \cdot J \cdot \nabla_{12},
$$

where

$$
V\left(\mathbf{r}_{1}-\mathbf{r}_{2}\right)=-\frac{\gamma^{2}}{2 \pi} \ln \left|\mathbf{r}_{1}-\mathbf{r}_{2}\right|
$$

Now we consider the Fourier transform of the function $V(\mathbf{r})$, where $\mathbf{r}=\mathbf{r}_{1}-\mathbf{r}_{2}$ :

$$
\begin{gathered}
V(\mathbf{r})=\int d \mathbf{k} \tilde{V}(\mathbf{k}) e^{i \mathbf{k} \cdot \mathbf{r}}, \\
\tilde{V}(\mathbf{k})=\frac{1}{(2 \pi)^{2}} \int d \mathbf{r} V(\mathbf{r}) e^{-i \mathbf{k} \cdot \mathbf{r}} .
\end{gathered}
$$

The Fourier transform of $V(\mathbf{r})$ is evaluated as follows:

$$
\begin{aligned}
\tilde{V}(\mathbf{k}) & =-\frac{\gamma^{2}}{(2 \pi)^{3}} \int d \mathbf{r} \ln |\mathbf{r}| e^{-i \mathbf{k} \cdot \mathbf{r}} \\
& =-\frac{\gamma^{2}}{(2 \pi)^{3}} \int_{0}^{\infty} r d r \int_{0}^{2 \pi} d \theta \ln r e^{-i k r \cos (\theta)} \\
& =-\frac{\gamma^{2}}{(2 \pi)} \int_{0}^{\infty} d r r \ln r J_{0}(k r) \\
& =-\frac{\gamma^{2}}{(2 \pi)^{2}}\left\{\left[\frac{r}{k} \ln r J_{1}(k r)\right]_{0}^{\infty}-\frac{1}{k} \int_{0}^{\infty} d r J_{1}(k r)\right\} .
\end{aligned}
$$

We have to evaluate the following limit:

$$
\lim _{r \rightarrow \infty} \frac{r}{k} \ln r J_{1}(k r) .
$$

In fact, for large $r$, this function oscillates with amplifying its absolute value. Therefore, we suppose that the limiting value of this function is zero. Alternatively, we insert a convergence factor:

$$
\lim _{\epsilon \rightarrow+0} \int_{0}^{\infty} d r r \ln r J_{0}(k r) e^{-\epsilon r}
$$

As a result, we have

$$
\widetilde{V}(\mathbf{k})=\frac{\gamma^{2}}{(2 \pi)^{2}} \frac{1}{k^{2}}
$$

The dependence of $\widetilde{V}(\mathbf{k}) \sim 1 / k^{2}$ is a typical behavior of Coulomb systems.

Now we evaluate each term an the right hand side of Eq. (43):

$$
\begin{aligned}
(A)= & \int_{0}^{t} d \tau U_{12}(\tau) \mathcal{L}_{12} U_{12}(-\tau) f_{1} f_{2} \\
= & -\int_{0}^{t} d \tau \mathbf{v}_{1}(t-\tau) \cdot \nabla_{12} f_{1} f_{2} \\
= & \frac{1}{\gamma} \int_{0}^{t} d \tau \int d \mathbf{k} \exp \left[i \mathbf{k} \cdot\left(\mathbf{r}_{1}-\mathbf{r}_{2}\right)-i \mathbf{k} \cdot \int^{\tau} d t^{\prime}\left\langle\mathbf{v}_{1}\right\rangle\right. \\
& \left.+i \mathbf{k} \cdot \int^{\tau} d t^{\prime}\left\langle\mathbf{v}_{2}\right\rangle\right] \widetilde{V}(k)(i \mathbf{k})^{\top} \cdot J \cdot \nabla_{12} f_{1} f_{2},
\end{aligned}
$$

$$
\begin{aligned}
(B)= & \int_{0}^{t} d \tau U_{12}(\tau) \mathcal{L}_{12} U_{12}(-\tau) g_{2}\left(\mathbf{r}_{1}, \mathbf{r}_{2} ; t\right) \\
= & -\int_{0}^{t} d \tau \mathbf{v}_{1}(t) \cdot \nabla_{12} g_{2}\left(\mathbf{r}_{1}, \mathbf{r}_{2} ; t\right) \\
= & \frac{1}{\gamma} \int_{0}^{t} d \tau \int d \mathbf{k} \exp \left[i \mathbf{k} \cdot\left(\mathbf{r}_{1}-\mathbf{r}_{2}\right)-i \mathbf{k} \cdot \int^{\tau} d t^{\prime}\left\langle\mathbf{v}_{1}\right\rangle\right. \\
& \left.+i \mathbf{k} \cdot \int^{\tau} d t^{\prime}\left\langle\mathbf{v}_{2}\right\rangle\right] \widetilde{V}(k)(i \mathbf{k})^{\top} \cdot J \cdot \nabla_{12} g_{2}\left(\mathbf{r}_{1}, \mathbf{r}_{2} ; t\right),
\end{aligned}
$$

$$
\begin{aligned}
(C)= & \int_{0}^{t} d \tau U_{12}(\tau) \int d \mathbf{r}_{3} \mathcal{L}_{13} U_{12}(-\tau) f_{1} g_{2}\left(\mathbf{r}_{2}, \mathbf{r}_{3} ; t\right) \\
= & \frac{1}{(2 \pi)^{2}} \gamma \int d \mathbf{R} \int d \mathbf{R}^{\prime} \int_{0}^{t} d \tau \int d \mathbf{k} \int d \mathbf{k}^{\prime} \\
& \times \exp \left[i \mathbf{k} \cdot\left(\mathbf{r}_{1}-\mathbf{R}^{\prime}\right)+i \mathbf{k}^{\prime} \cdot\left(\mathbf{r}_{2}-\mathbf{R}\right)\right. \\
& \left.-i \mathbf{k} \cdot \int^{\tau}\left\langle\mathbf{v}_{1}\right\rangle\right) d t^{\prime} \\
& \times \tilde{V}(k)\left[(i \mathbf{k})^{\top} \cdot J \cdot \nabla_{1} f_{1}\right] g_{2}\left(\mathbf{R}, \mathbf{R}^{\prime} ; t\right),
\end{aligned}
$$

$$
\begin{aligned}
(D)= & \int_{0}^{t} d \tau U_{12}(\tau) \int d \mathbf{r}_{3} \mathcal{L}_{23} U_{12}(-\tau) f_{2} g_{2}\left(\mathbf{r}_{1}, \mathbf{r}_{3} ; t\right) \\
= & \frac{1}{(2 \pi)^{2} \gamma} \int d \mathbf{R} \int d \mathbf{R}^{\prime} \int_{0}^{t} d \tau \int d \mathbf{k} \int d \mathbf{k}^{\prime} \\
& \times \exp \left[i \mathbf{k}^{\prime} \cdot\left(\mathbf{r}_{1}-\mathbf{R}\right)+i \mathbf{k} \cdot\left(\mathbf{r}_{2}-\mathbf{R}^{\prime}\right)-i \mathbf{k} \cdot \int^{\tau}\left\langle\mathbf{v}_{2}\right\rangle d t^{\prime}\right] \\
& \times \widetilde{V}(k)\left[(i \mathbf{k})^{\top} \cdot J \cdot \nabla_{2} f_{2}\right] g_{2}\left(\mathbf{R}, \mathbf{R}^{\prime} ; t\right) .
\end{aligned}
$$


The order estimate of these terms is as follows:

$$
(A) \sim \frac{1}{N}, \quad(B) \sim \frac{1}{N^{2}}, \quad(C) \sim \frac{1}{N}, \quad(D) \sim \frac{1}{N} .
$$

Therefore, we can neglect the term of $(B)$.

Then we obtain the following integral equation:

$$
\begin{aligned}
g_{2}\left(\mathbf{r}_{1}, \mathbf{r}_{2} ; t\right)= & q\left(\mathbf{r}_{1}, \mathbf{r}_{2} ; t\right)+\int d \mathbf{R} \int d \mathbf{R}^{\prime} \\
& \times K\left(\left\{\mathbf{r}_{1}, \mathbf{r}_{2}\right\},\left\{\mathbf{R}, \mathbf{R}^{\prime}\right\}\right) g_{2}\left(\mathbf{R}, \mathbf{R}^{\prime} ; t\right),
\end{aligned}
$$

where

$$
\begin{aligned}
q\left(\mathbf{r}_{1}, \mathbf{r}_{2} ; t\right)= & \frac{1}{\gamma} \int_{0}^{t} d \tau \int d \mathbf{k} \exp \left[i \mathbf{k} \cdot\left(\mathbf{r}_{1}-\mathbf{r}_{2}\right)-i \mathbf{k} \cdot \int^{\tau} d t^{\prime}\left\langle\mathbf{v}_{1}\right\rangle\right. \\
& \left.+i \mathbf{k} \cdot \int^{\tau} d t^{\prime}\left\langle\mathbf{v}_{2}\right\rangle\right] \tilde{V}(k)(i \mathbf{k})^{\top} \cdot J \cdot \nabla_{12} f_{1} f_{2},
\end{aligned}
$$

and

$$
\begin{aligned}
K\left(\left\{\mathbf{r}_{1}, \mathbf{r}_{2}\right\},\left\{\mathbf{R}, \mathbf{R}^{\prime}\right\}\right) & =\frac{1}{(2 \pi)^{2} \gamma} \int_{0}^{t} d \tau \int d \mathbf{k} \int d \mathbf{k}^{\prime} \exp \left[i \mathbf{k} \cdot\left(\mathbf{r}_{1}-\mathbf{R}^{\prime}\right)\right. \\
& +i \mathbf{k}^{\prime} \cdot\left(\mathbf{r}_{2}-\mathbf{R}\right)-i \mathbf{k} \cdot \int^{\tau}\left(\left\langle\mathbf{v}_{1}\right\rangle d t^{\prime}\right] \\
& \times \tilde{V}(k)(i \mathbf{k})^{\top} \cdot J \cdot \nabla_{1} f_{1}+\frac{1}{(2 \pi)^{2} \gamma} \int_{0}^{t} d \tau \int d \mathbf{k} \int d \mathbf{k}^{\prime} \\
& \times \exp \left[i \mathbf{k}^{\prime} \cdot\left(\mathbf{r}_{1}-\mathbf{R}\right)+i \mathbf{k} \cdot\left(\mathbf{r}_{2}-\mathbf{R}^{\prime}\right)\right. \\
& \left.-i \mathbf{k} \cdot \int^{\tau}\left\langle\mathbf{v}_{2}\right\rangle d t^{\prime}\right] \tilde{V}(k)(i \mathbf{k})^{\top} \cdot J \cdot \nabla_{2} f_{2} .
\end{aligned}
$$

The function $K\left(\left\{\mathbf{r}_{1}, \mathbf{r}_{2}\right\},\left\{\mathbf{R}, \mathbf{R}^{\prime}\right\}\right)$ is called the integral kernel of the integral equation. This integral equation takes the form of the Fredholm integral equation of the second kind. Thus, how to solve it is known [33]. Now, for brevity, we set $\mathbf{x}$ $=\left(\mathbf{r}_{1}, \mathbf{r}_{2}\right)$ and $\mathbf{y}=\left(\mathbf{R}, \mathbf{R}^{\prime}\right)$. The integral equation, which should be solved, is

$$
g_{2}(\mathbf{x} ; t)=q(\mathbf{x} ; t)+\lambda \int d \mathbf{y} K(\mathbf{x}, \mathbf{y}) g_{2}(\mathbf{y} ; t) .
$$

If the required conditions are satisfied, this integral equation is solved as

$$
g_{2}(\mathbf{x} ; t)=q(\mathbf{x} ; t)+\int d \mathbf{y} \Xi\left(\mathbf{x}, \mathbf{y} ; \lambda_{0}\right) q(\mathbf{y} ; t) .
$$

The function $\Xi(\mathbf{x}, \mathbf{y} ; \lambda)$ is called the resolvent. $\lambda_{0}$ is chosen to make the series convergent. For large $N$, the kernel $K(\mathbf{x}, \mathbf{y})$ is $\sim 1 / N$. Thus, for large $N$, we can take as $\lambda_{0}=1$. Therefore, if the kernel is bounded, for large $N$, we get a convergent series. The resolvent is given by

$$
\Xi(\mathbf{x}, \mathbf{y} ; \lambda)=\frac{D(\mathbf{x}, \mathbf{y} ; \lambda)}{D(\lambda)},
$$

where

$$
\begin{aligned}
D(\lambda)= & 1-\lambda \int d \mathbf{s} K(\mathbf{s}, \mathbf{s})+\frac{\lambda^{2}}{2 !} \iint d \mathbf{s}_{1} d \mathbf{s}_{2} K\left(\begin{array}{ll}
\mathbf{s}_{1} & \mathbf{s}_{2} \\
\mathbf{s}_{1} & \mathbf{s}_{2}
\end{array}\right)+\cdots \\
& +\frac{(-\lambda)^{p}}{p !} \int \cdots \int d \mathbf{s}_{1} \cdots d \mathbf{s}_{p} K\left(\begin{array}{llll}
\mathbf{x} & \mathbf{s}_{1} & \cdots & \mathbf{s}_{p} \\
\mathbf{y} & \mathbf{s}_{2} & \cdots & \mathbf{s}_{p}
\end{array}\right) \\
& +\cdots
\end{aligned}
$$

$$
\begin{aligned}
D(\mathbf{x}, \mathbf{y} ; \lambda)= & K(\mathbf{x}, \mathbf{y})-\lambda \int d \mathbf{s} K\left(\begin{array}{ll}
\mathbf{x} & \mathbf{s} \\
\mathbf{y} & \mathbf{s}
\end{array}\right) \\
& +\frac{\lambda^{2}}{2 !} \iint d \mathbf{s}_{1} d \mathbf{s}_{2} K\left(\begin{array}{lll}
\mathbf{x} & \mathbf{s}_{1} & \mathbf{s}_{2} \\
\mathbf{y} & \mathbf{s}_{2} & \mathbf{s}_{2}
\end{array}\right)+\cdots \\
& +\frac{(-\lambda)^{p}}{p !} \int \cdots \int d \mathbf{s}_{1} \cdots d \mathbf{s}_{p} K\left(\begin{array}{cccc}
\mathbf{x} & \mathbf{s}_{1} & \cdots & \mathbf{s}_{p} \\
\mathbf{y} & \mathbf{s}_{2} & \cdots & \mathbf{s}_{p}
\end{array}\right) \\
& +\cdots
\end{aligned}
$$

and

$$
K\left(\begin{array}{llll}
\mathbf{s}_{1} & \mathbf{s}_{2} & \cdots & \mathbf{s}_{p} \\
\mathbf{t}_{1} & \mathbf{t}_{2} & \cdots & \mathbf{t}_{p}
\end{array}\right)=\left|\begin{array}{cccc}
K\left(\mathbf{s}_{1}, \mathbf{t}_{1}\right) & K\left(\mathbf{s}_{1}, \mathbf{t}_{2}\right) & \cdots & K\left(\mathbf{s}_{1}, \mathbf{t}_{p}\right) \\
K\left(\mathbf{s}_{2}, \mathbf{t}_{1}\right) & K\left(\mathbf{s}_{2}, \mathbf{t}_{2}\right) & \cdots & K\left(\mathbf{s}_{2}, \mathbf{t}_{p}\right) \\
\cdots & \cdots & \cdots & \cdots \\
K\left(\mathbf{s}_{p}, \mathbf{t}_{1}\right) & K\left(\mathbf{s}_{p}, \mathbf{t}_{2}\right) & \cdots & K\left(\mathbf{s}_{p}, \mathbf{t}_{p}\right)
\end{array}\right| .
$$

Thus, a derived kinetic equation is

$$
\frac{\partial f_{1}}{\partial t}+\left\langle\mathbf{v}_{1}\right\rangle \cdot \nabla_{1} f_{1}=\int d \mathbf{r}_{2} \mathcal{L}_{12} g_{2}\left(\mathbf{r}_{1}, \mathbf{r}_{2} ; t\right),
$$

where

$$
\begin{aligned}
g_{2}\left(\mathbf{r}_{1}, \mathbf{r}_{2} ; t\right)= & q\left(\mathbf{r}_{1}, \mathbf{r}_{2} ; t\right)+\int d \mathbf{R} \int d \mathbf{R}^{\prime} \\
& \times \Xi\left(\left\{\mathbf{r}_{1}, \mathbf{r}_{2}\right\},\left\{\mathbf{R}, \mathbf{R}^{\prime}\right\} ; \lambda_{0}\right) q\left(\mathbf{R}, \mathbf{R}^{\prime} ; t\right) .
\end{aligned}
$$

This kinetic equation for point vortex system is an analog of the Balescu-Lenard equation in plasma physics. Unlike the Balescu-Lenard equation for plasmas, the collision term obtained does not have the dielectric function, but has additional terms compared with the Landau collision term. We note an important point which manifests a difference between point vortex systems and plasma systems. The kernel has the factor $1 / N$, which comes from the circulation $\gamma$ $=\Gamma / N$. Thus, the resolvent is expanded in terms of the $1 / N$-factor. The integral is just the Fourier transform. Therefore, the integral is order of 1. In Eq. (64), the second term on the right-hand side is smaller than the first term as the order $O(1 / N)$. Therefore, for large $N$, we obtain

$$
g_{2}\left(\mathbf{r}_{1}, \mathbf{r}_{2} ; t\right) \approx q\left(\mathbf{r}_{1}, \mathbf{r}_{2} ; t\right) .
$$

With this result, the collision term is reduced to the Landau collision term: i.e., Eq. (38). Therefore, for point vortex systems, the Balescu-Lenard collision term is reduced to the 
Landau collision term, because of large $N$-effect: i.e., the absence of the dielectric function. This is a big difference between point vortex systems and plasma systems. The screening effect does not appear in point vortex systems, while the screening effect is important for the BalescuLenard equation for plasmas. On the Balescu-Lenard collision term in plasma physics, see Ref. [32] for comparison.

In the above paragraph, we showed how to solve the integral equation (63) formally. But the required conditions have not yet been checked. Let us look at the required conditions. First, to show that the operator $T$,

$$
T g_{2}=\int d \mathbf{y} K(\mathbf{x}, \mathbf{y}) g_{2}(\mathbf{y}),
$$

is bounded is important. The integral equation is symbolically given by

$$
(1-\lambda T) g_{2}=q .
$$

The formal solution is obtained by the expansion

$$
g_{2}=\sum_{n=0}^{\infty}(\lambda T)^{n} q
$$

The Fredholm theory for integral equation basically uses the boundedness of the operator and the expansion: i.e., Eq. (74). This expansion is assured by the boundedness of the operator $T$. The expansions of Eqs. (66) and (67) are also due to the boundedness of the operator $T$. As the second point, unlike usual Fredholm integral equations, the integral domain in eq. (63) is infinite. If the integral domain is finite and the kernel is bounded, the boundedness of operators is easily shown. For our case, since the integral domain is infinite, we should treat the operator $T$ carefully. These two points-i.e., the boundedness of the operator $T$ and the infinite integral domain - should be checked and be treated in a rigorous way. However, in this paper, we do not pursue a rigorous discussion. These problems are reserved for mathematical physicists.

\section{CONCLUDING REMARKS}

We have derived a kinetic equation for point vortex systems in an infinite plane. The kinetic equations derived are analog of the Landau equation and the Balescu-Lenard equation. Equation (39) coincides with the result of Chavanis $[15,27]$. Equation (39) possesses several interesting properties, which were shown in Ref. [15]. The Balescu-Lenard equation (69) is a new kinetic equation. Furthermore, we have shown that for large $N$, the Balescu-Lenard equation (69) is reduced to the Landau equation (39). Therefore, we can conclude that for point vortex systems in an infinite plane, without symmetrical restriction (such as axisymmetric and unidirectional flows), the most generalized kinetic equation is Eq. (39).

The following point would be the interesting point of the derived kinetic equation, (39): The interaction among point vortices is long range-i.e., logarithmic. In addition, the derived kinetic equation is analogous to the Landau equation for 3D plasmas. However, the collision term for point vortex systems may not diverge. In Ref. [27], for the axisymmetric case, the collision term does not diverge. This is a symptom of the nondivergence of the collision term for the Landau equation. The reason of this is that the difference between the integration of the collision term-i.e., Eq. (38) - and that of the Landau collision term for 3D plasmas. The former has the integration with respect to $\mathbf{r}_{2}$ (i.e., position), while the latter has the integration with respect to $\mathbf{r}_{2}$ and $\mathbf{v}_{2}$ (i.e., velocity).

For the point vortex systems, the expression of the energy spectrum was derived for the system in an infinite plane [34] and for the system in a circular domain [35]. The energy spectrum is closely related to the diffusion coefficient [36]. We will be able to compare the theory of Ref. [36] with the kinetic theory in this paper.

Another interesting point is the following: our kinetic theory is not directly connected to Onsager's temperature. As shown in Ref. [37] for two-sign point vortex systems, Onsager's temperature affects nonequilibrium properties: i.e., decaying process. To find this connection leads to understand nonequilibirum properties: i.e., classification of nonequilibrium processes.

The most interesting problem, which we would like to attack with Eq. (39), is a decaying property of vortex crystals in non-neutral plasmas. Unfortunately, Eq. (39) is for the system in an infinite plane, not for the system in a circular domain. Thus, it is not for an experimental situation. But Eq. (39) surely captures the nature of phenomena for the system in a circular domain in some extent. Many experimental results show that the vortex crystals are quasi-stationary states. To analyze quasistationary states, recent advances in longrange interaction systems would be some hints for us, such as a study of the Hamiltonian mean field (HMF) model. The HMF model exhibits a slow decay, in which the state is stuck in a quasistationary state, as well as in point vortex systems. For the HMF model, the slow decay is analyzed by the Vlasov equation. The quasistationary state is very near to a stable solution of the Vlasov equation. The estimate of some quantities-i.e., the algebraic decay and the tail of the velocity distribution function, etc,- -is carried out [38] and is tested by a numerical simulation [39]. Their analysis would be useful for our problems. However, to this end, we should know the behavior of the 2D Euler equation, instead of the Vlasov equation for the usual kinetic theory of particle systems.

\section{ACKNOWLEDGMENTS}

The author thanks Professor H. Tomita for continuous encouragement and Professor Y. Kiwamoto for introducing vortex dynamics. The author is grateful to an anonymous referee for pointing out Ref. [27] and numerous other helpful suggestions. 
[1] A. J. Chorin, Vorticity and Turbulence (Springer-Verlag, Berlin, 1994).

[2] P. K. Newton, The N-Vortex Problem: Analytical Techniques (Springer, New York, 2000).

[3] H. von Helmholtz, Philos. Mag. 4, 485 (1858).

[4] G. R. Kirchhoff, Vorlesungen über Mathematische Physik (Teubner, Leipzig, 1876), Vol. I.

[5] L. Onsager, Nuovo Cimento, Suppl. 6, 279 (1949).

[6] G. L. Eyink and K. R. Sreenivasan, Rev. Mod. Phys. 78, 87 (2006).

[7] S. Kida, J. Phys. Soc. Jpn. 39, 1395 (1975).

[8] G. Joyce and D. Montgomery, J. Plasma Phys. 10, 107 (1973).

[9] Y. B. Pointin and T. S. Lundgren, Phys. Fluids 19, 1459 (1976).

[10] T. S. Lundgren and Y. B. Pointin, J. Stat. Phys. 17, 323 (1977).

[11] C. E. Seyler Jr., Phys. Fluids 19, 1336 (1976).

[12] P. A. Smith and T. M. O’Neil, Phys. Fluids B 2, 2961 (1990).

[13] A. C. Ting, H. H. Chen, and Y. C. Lee, Physica D 26, 37 (1987)

[14] H. Marmanis, Proc. R. Soc. London, Ser. A 454, 587 (1998).

[15] P. H. Chavanis, Phys. Rev. E 64, 026309 (2001).

[16] C. F. Driscoll and K. S. Fine, Phys. Fluids B 2, 1359 (1990).

[17] K. S. Fine, A. C. Cass, W. G. Flynn, and C. F. Driscoll, Phys. Rev. Lett. 75, 3277 (1995).

[18] D. Z. Jin and D. H. E. Dubin, Phys. Rev. Lett. 80, 4434 (1998).

[19] D. Schecter, D. H. E. Dubin, K. S. Fine, and C. F. Driscoll, Phys. Fluids 11, 905 (1999).

[20] Y. Kiwamoto, K. Ito, A. Sanpei, and A. Mohri, Phys. Rev. Lett. 85, 3173 (2000).

[21] A. Sanpei, Y. Kiwamoto, K. Ito, and Y. Soga, Phys. Rev. E 68, 016404 (2003).
[22] Y. Soga, Y. Kiwamoto, A. Sanpei, and J. Aoki, Phys. Plasmas 10, 3922 (2003).

[23] R. Kawahara and H. Nakanishi, J. Phys. Soc. Jpn. 75, 054001 (2006).

[24] R. Kawahara and H. Nakanishi, J. Phys. Soc. Jpn. 78, 074001 (2007).

[25] M. M. Sano, Y. Yatsuyanagi, Y. Yoshida, and H. Tomita, J. Phys. Soc. Jpn. 76, 064001 (2007).

[26] P. H. Chavanis and M. Lemou, e-print arXiv:cond-mat/ 0703023.

[27] P. H. Chavanis (unpublished).

[28] N. N. Bogoliubov, J. Phys. (Moscow) 10, 257 (1946).

[29] M. Born and H. S. Green, Proc. R. Soc. London, Ser. A 188, 10 (1946).

[30] J. G. Kirkwood, J. Chem. Phys. 14, 180 (1946).

[31] J. Yvon, La théorie Statistique des fluides et l'équation d'état, Actualités scientifiques et industrielles, No. 203 (Hermann, Paris, 1935).

[32] R. Balescu, Statistical Dynamics: Matter out of equilibrium (Imperial College Press, London, 1997).

[33] R. Courant and D. Hilbert, Methoden der Mathematischen Physik, 2nd ed. (Springer-Verlag, Berlin, 1931).

[34] E. A. Novikov, Sov. Phys. JETP 41, 937 (1976).

[35] T. Yoshida and M. M. Sano, J. Phys. Soc. Jpn. 74, 587 (2005).

[36] J. B. Taylor and B. McNamara, Phys. Fluids 14, 1492 (1971).

[37] Y. Yatsuyanagi, Y. Kiwamoto, H. Tomita, M. M. Sano, T. Yoshida, and T. Ebisuzaki, Phys. Rev. Lett. 94, 054502 (2005).

[38] F. Bouchet and T. Dauxois, Phys. Rev. E 72, 045103(R) (2005).

[39] Y. Y. Yamaguchi, F. Bouchet, and T. Dauxois, J. Stat. Mech.: Theory Exp. 2007, P01020. 